\title{
Rickets presenting as gross motor delay in twin girls
}

\author{
Charushree Prasad MD, Elizabeth Cummings MD
}

Cite as: CMAJ 2018 May 7;190:565-8. doi: 10.1503/cmaj.170962

$\mathbf{T}$ win 12-month-old girls underwent neurologic assessment for poor tone and gross motor delay. The infants first presented to their pediatrician at six months of age, when they were unable to roll or sit. At seven months, physiotherapy assessment showed that the twins were at the fifth percentile for age in gross motor function. They began to roll and sit unassisted at seven months, but they could not crawl or stand. Their parents stated that the girls seemed uncomfortable when encouraged to stand during physiotherapy. Other developmental milestones were unaffected.

The infants had been born at term by cesarean delivery because of failure-of-progression, following a healthy dichorionic diamniotic twin pregnancy. They were formula-fed and started taking homogenized milk at one year of age, with a well-balanced diet. One of the twins had a urinary tract infection at six months of age, with no sequelae. There was no history of seizures or muscle spasms. The twins took no prescribed or over-the-counter medicines, and no herbal supplements. Before visiting the neurologist, one twin had undergone investigation for several unexplained episodes of vomiting, each of which had resolved within a day. Both parents were of Maritime French-Canadian (Acadian) heritage from the same geographic area, but were not known to be related.

On physical examination, both twins were between the 10th and 25th percentiles for weight and head circumference, but below the 3rd percentile for length. Dysmorphic features were noted: frontal bossing with the appearance of hypertelorism and proptosis, a flat nasal bridge and an open anterior fontanelle. The infants had poor generalized tone and remarkable weakness, but their reflexes were normal with no clonus or fasciculations. There were no other concerning findings on physical examination.

Owing to the dysmorphic features, gross motor delay and recurrent vomiting episodes, the pediatrician considered a disorder of carbohydrate metabolism or congenital myopathy. A series of investigations including venous blood gas, lactate, liver and renal function tests and creatine kinase (for myopathy) showed normal results, other than very elevated alkaline phosphatase levels (3428 and 2986 [normal 145-320] mU/L). Owing to this elevation, the endocrinology service was consulted and recommended investigations for rickets. Calcium, corrected for

\section{KEY POINTS}

- A diagnosis of rickets should be considered in infants and young children who present with failure to thrive, weakness or developmental delay that primarily involves gross motor skills.

- Although vitamin D deficiency is the most common cause of rickets, genetic defects should be considered when 25-hydroxy vitamin D levels are normal, or the dietary history does not suggest poor intake of vitamin D.

- Canadian physicians should be aware of the higher risk of 1-a-hydroxylase deficiency in the French-Canadian populations of Quebec and the Maritime provinces.

slightly high albumin levels, was low (1.25 and 1.20 [normal 2.12.7] $\mathrm{mmol} / \mathrm{L}$ ] for each twin. In addition, phosphate values were low, at 1.1 and 1.3 (normal $1.5-2.2$ ) mmol/L.

Radiography of the twins' knees showed metaphyseal cupping and fraying of the tibia, fibula and distal femurs, with delayed ossification of the epiphyses (Figure 1). Radiography of the wrists showed similar fraying and cupping, typical of rickets (Figure 2).

Nutritional vitamin $D$ deficiency could not explain the rickets given the normal levels of 25-hydroxyvitamin D (67.1 and 67.8 [normal 50-200] nmol/L), but both twins had very low levels of 1,25-dihydroxyvitamin D (24 and 21 [normal 60-159] pmol/L. We requested parathyroid hormone testing, but it was not performed.

A diagnosis of 1-a-hydroxylase deficiency (also known as vitamin D-dependent rickets type 1) was confirmed by molecular testing that showed a homozygous mutation in the CYP271B1 gene.

Treatment with alfacalcidol (1- $\alpha$-hydroxycholecalciferol) and calcium was started and brought steady improvement, and both twins showed normal serum calcium levels after two months. Radiography four months later showed improvement in the condition of the twins' knees and wrists, but persistent rachitic changes. After one year of treatment, radiography showed complete healing of their bones, and their motor development was normal.

Their parents later discovered that they were distantly related and there was a history of possible rickets in multiple family members. 


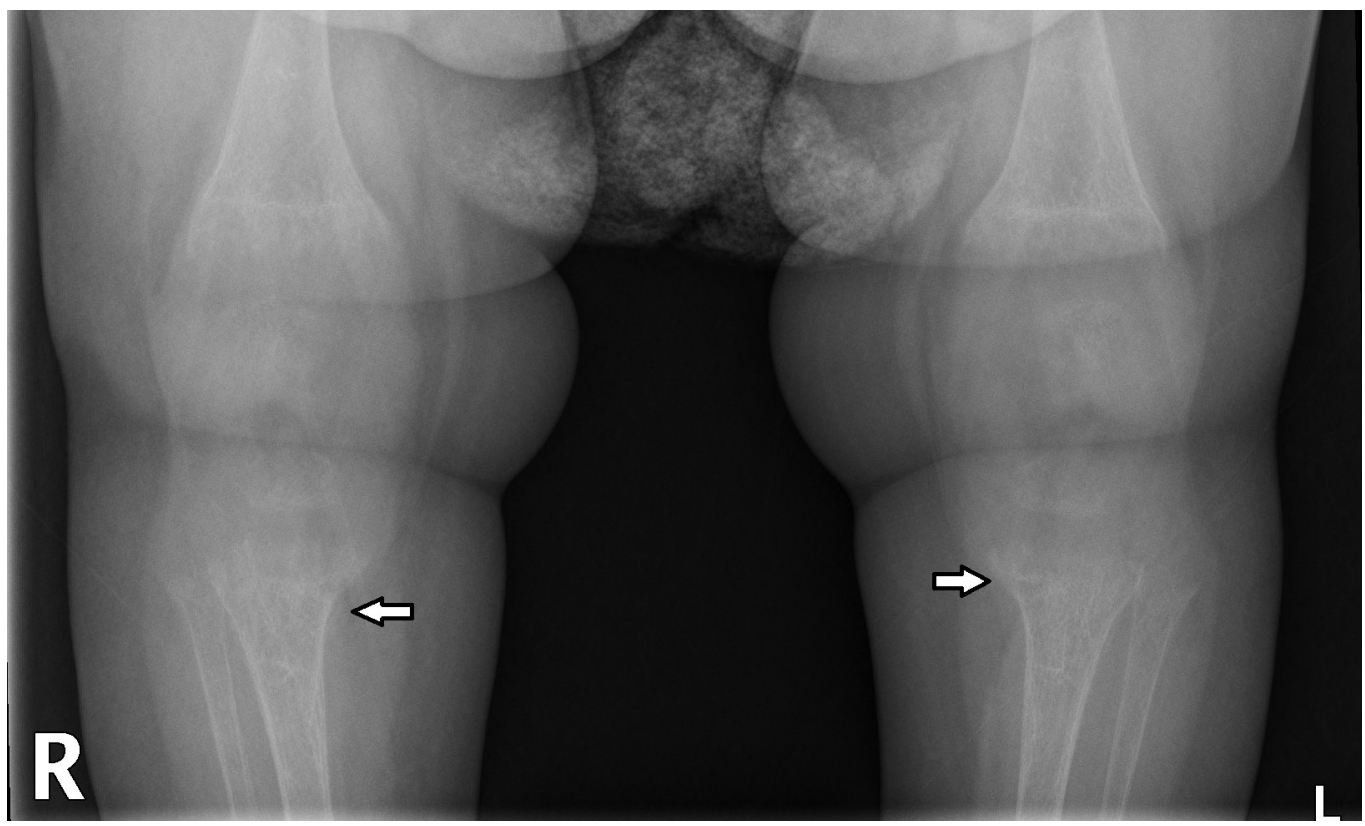

Figure 1: Bilateral radiographic imaging of the knees of a 12-month-old girl showing metaphyseal cupping and flaring consistent with rickets (arrows). The infant and her twin sister presented with poor tone and gross motor delay.

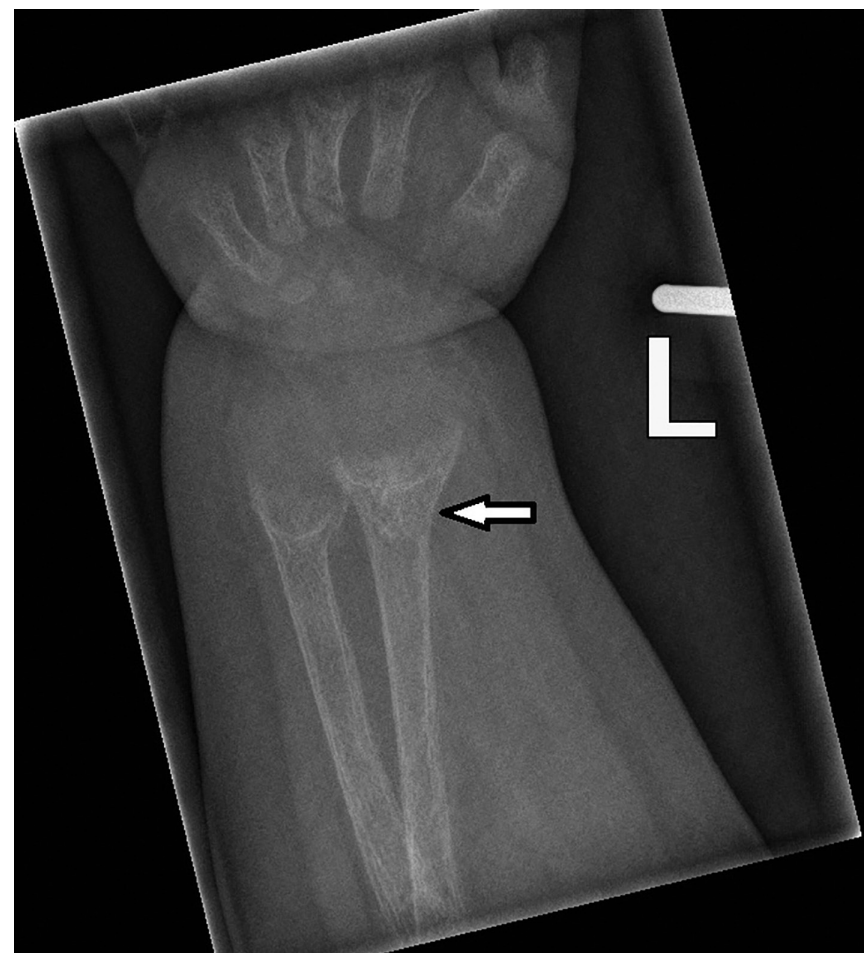

Figure 2: Radiographic image of the left wrist of one of the twins, showing typical rachitic changes of cupping and flaring (arrow).

\section{Discussion}

Gross motor delay, weakness and hypotonia are a common clinical presentation of neuromuscular disorders, such as congenital myopathies and cerebral palsy. Rickets can also present with these symptoms, although it is not usually listed in clinical texts as a potential cause. Rickets should be considered if the clinical history does not fit with typical neuromuscular disorders. ${ }^{1}$ Rickets is easily excluded and, as seen in these patients' cases, a delay in treatment can lead to substantial gross motor delay, bony changes, pain and poor growth (Figure 3). Low calcium levels likely contribute to muscle dysfunction, while bone weakness leads to discomfort and reduced weight-bearing, with subsequent delayed motor skills.

The pathophysiology of rickets involves deficiency of calcium or phosphate in growing bone, resulting in decreased bone matrix production and deficient growth plate mineralization, the hallmarks of the disease (Box 1$).{ }^{1}$ The adult equivalent is osteomalacia.

Calcium-deficient rickets is most commonly related to poor nutritional intake or malabsorption of calcium or vitamin D. Single-gene disorders (such as mutations in the genes encoding 1-a-hydroxylase, 25-hydroxylase or the vitamin D receptor) are less common causes of rickets. ${ }^{2}$ Because vitamin D facilitates intestinal and renal absorption of calcium, decreased vitamin $D$ action leads to calcium deficits. To maintain blood calcium levels, parathyroid hormone is increased and acts on bones to disrupt bone homeostasis, causing the release of calcium. The accumulation of demineralized endochondral matrix at the growth plate results in the epiphyseal cupping and flaring seen on radiography. ${ }^{1}$

Vitamin D status is dependent on intestinal absorption of vitamin D2 (ergocalciferol) or vitamin D3 (cholecalciferol), in addition to the skin's production of vitamin D3 in response to ultraviolet radiation (sunlight). ${ }^{3}$ Vitamin $\mathrm{D}$ remains inactive until it is converted to 25-hydroxyvitamin D (calcidiol) by the liver, and then to 1,25-dihydroxyvitamin $D$ (calcitriol) by the enzyme 1-a-hydroxylase in the renal proximal tubule. ${ }^{1}$ Deficiency of 1-a-hydroxylase leads to hypocalcemia and mildly reduced phosphate, with secondary elevation in parathyroid hormone. In this autosomal recessive form of rickets, calcidiol is normal while calcitriol is low (as seen in these patients' cases) (Box 2). This 
biochemical finding is in contrast to hypophosphatemic rickets, most often characterized by renal phosphate wasting, in which calcium is normal but phosphate is very low, and parathyroid hormone is usually normal. ${ }^{1}$

The clinical presentation of both calcium- and phosphatedeficient rickets (Figure 3) can be similar and include poor growth, hypotonia and changes to the bones and teeth. ${ }^{1}$ Patients with advanced cases can present with seizures, fractures and delayed motor milestones. ${ }^{4}$ Typical radiographic changes include osteopenia, and flaring and cupping of the metaphyses (Figures 1 and 2); flared rib ends can cause the "rachitic rosary" (Figure 3).

Vitamin D deficiency is common and is currently a prominent public health topic because vitamin D is linked not only to bone health, but also to autoimmune disorders, immune function and malignant disease. ${ }^{5}$ Risk factors for vitamin D deficient rickets in Canada include severe maternal vitamin D deficiency, lack of sun exposure, northern latitude residence, immigrant status, and

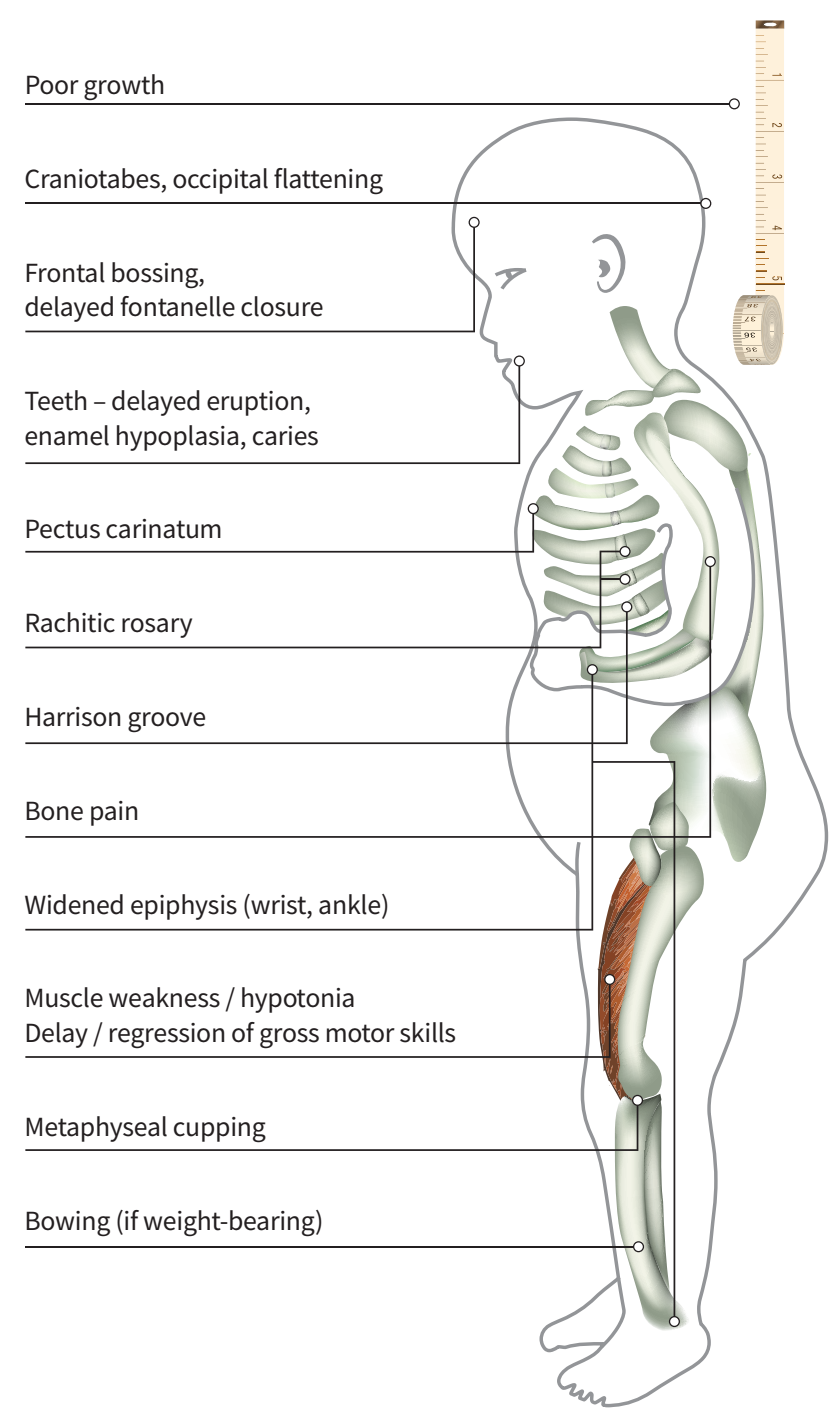

Figure 3: Clinical manifestations of rickets that involve musculoskeletal and neurologic changes.

darker skin pigmentation. ${ }^{4}$ When patients have no risk factors or show no improvement after starting vitamin D therapy, a genetic cause should be suspected. One should consider, however, the possibility of poor adherence because vitamin D supplements are over-the-counter products, which may not be reimbursed by drug plans.

There is a known association with genetic rickets in Canada owing to two CYP27B1 founder populations: French Canadians in the Charlevoix-Saguenay-Lac-Saint Jean region of Quebec, with a carrier rate as high as 1:26, and the Acadian population in the Maritimes, with an estimated carrier frequency of $1: 60 .^{6,7}$ French colonization of Maritime Canada in the 1600 s led to the origin of the Acadian population, now spread over New Brunswick, Prince Edward Island, Nova Scotia, and the northeastern states of the United States. ${ }^{8}$ Acadian families may be unaware of the increased risk of rickets. The twins in this case were of Acadian ancestry and had homozygous mutations in the CYP27B1 gene that encodes 1-a-hydroxylase.

Nutritional rickets is treated with high doses of the usual form of vitamin D, cholecalciferol. ${ }^{5}$ However, this treatment is ineffective in 1-a-hydroxylase deficiency because the body cannot convert cholecalciferol to active vitamin D1. Therefore, supplementation with a 1-a-hydroxylated form of vitamin D, either calcitriol or alfacalcidol, is essential. Alfacalcidol, available in Canada as a liquid, is the preferred option for young children. Calcium levels

Box 1: Comparison of laboratory values in calcium- and phosphate-deficient rickets ${ }^{1}$

\begin{tabular}{|lll|}
\hline $\begin{array}{l}\text { Laboratory value } \\
\text { Calcium }\end{array}$ & $\begin{array}{c}\text { Calcium-deficient } \\
\text { rickets }\end{array}$ & $\begin{array}{c}\text { Phosphate- } \\
\text { deficient rickets }\end{array}$ \\
\hline Phosphate & Decreased or normal & Normal \\
\hline $\begin{array}{l}\text { Alkaline } \\
\text { phosphatase }\end{array}$ & Increased & Decreased \\
\hline $\begin{array}{l}\text { Parathyroid } \\
\text { hormone }\end{array}$ & Increased & Increased \\
\hline
\end{tabular}

Box 2: Comparison of laboratory values in calciumdeficient rickets caused by nutritional vitamin $D$ deficiency and 1- $\alpha$-hydroxylase deficiency ${ }^{1}$

Laboratory value
Calcium
Phosphate
25-hydroxyvitamin D
1,25-dihydroxyvitamin D

Parathyroid hormone

\section{Nutritional vitamin D deficiency}

Normal or decreased

Normal or decreased

Decreased

Decreased, normal or increased, depending on severity

Increased
1- $\alpha$ hydroxylase deficiency

Decreased

Decreased

Normal

Decreased 
should begin to improve with supplementation within several days, and the rachitic changes can resolve within one year.

Management of 1- $\alpha$-hydroxylase deficiency rickets should occur in consultation with a specialist. Adequate calcium must be provided initially to avoid hypocalcemia related to "hungry bone syndrome," in which the healing bone avidly takes up calcium. Doses are adjusted according to biochemical and radiographic response, with the goals of normalizing serum calcium, alkaline phosphatase and parathyroid hormone, resolution of rachitic changes, and normalization of growth. When monitoring alkaline phosphatase, pediatric reference ranges should be used, since the lower adult ranges may be misleading. Adverse effects of treatment include hypercalcemia and hypercalciuria, which can lead to nephrocalcinosis requiring monitoring of urine calcium-to-creatinine ratio and kidney function, in addition to periodic renal ultrasonography. ${ }^{1,3}$

These cases show that rickets can present with substantial neuromuscular dysfunction and weakness, rather than fractures or growth concerns.

\section{References}

1. Root AW, Diamond FB Jr. Disorders of mineral homeostasis in children and adolescents. In: Sperling MA, editor. Pediatric endocrinology. 4th ed. Philadelphia: Saunders; 2014;779-87.

2. Malloy PJ, Feldman D. Genetic disorders and defects in vitamin D action. Endocrinol Metab Clin North Am 2010;39:333-46, table of contents.

3. Glorieux FH, Pettifor JM. Vitamin D/dietary calcium deficiency rickets and pseudo-vitamin D deficiency rickets. Bonekey Rep 2014;3:524.

4. Ward LM, Gaboury I, Ladhani M, et al. Vitamin D-deficiency rickets among children in Canada. CMAJ 2007;177:161-6.

5. Godel JC. Vitamin D supplementation: recommendations for Canadian mothers and infants. Paediatr Child Health 2007;12:583-98.

6. Portale AA, Miller WL. Hereditary rickets revealed. Kidney Int 1998;54:1762-4.

7. Portale AA, Miller WL. Human 25-hydroxyvitamin D-1- $\alpha$-hydroxylase: cloning, mutations, and gene expression. Pediatr Nephrol 2000;14:620-5.

8. Labuda M, Labuda D, Korab-Laskowska M, et al. Linkage disequilibrium analysis in young populations: pseudo-vitamin-D-defiency rickets and the founder effect in French Canadians. Am J Hum Genet 1996;59:633-43.

\section{Competing interests: None declared.}

This article has been peer reviewed.

The authors have obtained patient consent.

Affiliations: Department of Paediatrics (Prasad, Cummings), IWK Health Centre; Division of Endocrinology (Cummings), Department of Pediatrics, Dalhousie University, Halifax, NS
Contributors: Both authors made substantial contributions to this work and meet the criteria for authorship. Elizabeth Cummings conceived the idea for the report. Both authors reviewed the data from the cases and the literature, and analyzed and synthesized the information. Charushree Prasad wrote the first draft, and Elizabeth Cummings provided cri- tical revision. Both authors approve this final version of the manuscript and agree to be held accountable for all aspects of the work.

Correspondence to: Elizabeth Cummings, elizabeth.cummings@dal.ca 